\title{
Inter-laboratory test on drug residues in water intended for human consumption
}

\author{
J.S. Py ${ }^{\star}$, C. Rosin, N. Rimlinger, and J.F. Munoz \\ ANSES-Laboratoire d'hydrologie de Nancy, 40 rue Lionnois, 54000 Nancy, France
}

Received: 5 May 2012 / Accepted: 13 June 2012

\begin{abstract}
In June 2009, the Laboratory in hydrology of Nancy (Ansès-LHN, Nancy) conducted an exploratory inter-laboratory proficiency tests (ILT) test in collaboration with the AQUAREF network to identify laboratory practices and estimate inter-laboratory uncertainty for the compounds under study. There were a total of 12 compounds belonging to six families (hormones, antibiotics, non-steroidal antiinflammatory drugs, beta blockers, antipsychotics and others) including one compound used in veterinary medicine. It was the first ILT conducted in France on water intended for human consumption. Thirty-one laboratories participated, 4 of which were located abroad.
\end{abstract}

Keywords: Interlaboratory test; hydrology; uncertainty; drugs; human consumption

\section{Introduction}

In accordance with French regulations and the recommendations of the French Accreditation Committee (COFRAC) in French Standard NF EN 17025, laboratories are required to take part in inter-laboratory proficiency tests (ILPT). The goal of these ILPT is to verify the laboratories' analytical proficiency for given parameters and methods. Exploratory ILT aim to provide a snapshot of the profession at a point in time $t$ in preparation for a method's standardisation.

In this context, Ansès's Laboratory in hydrology of Nancy, in collaboration with the AQUAREF network, organised an inter-laboratory test on drug residues in june 2009. After consultation with the various participants, a list of 12 compounds was established. This list included various families of human compounds (hormones, antibiotics, non-steroidal anti-inflammatory drugs, beta blockers, antipsychotics and other) and Tylosin, an antibiotic used specifically in veterinary medicine. Thirty-one laboratories participated in this test, 4 of which were located abroad. The goals of this test on drug residues in water are (the following):

- to compile a list of analytical laboratories that had started developing analysis techniques;

- to obtain information related to analytical performance and uncertainties;

- to stimulate inter-laboratory cooperation on extraction and detection-quantitation methods;

- to draw up legislative and regulatory texts.

^ Correspondence: Jean-Sebastien.PY@anses.fr

\section{The samples}

Five samples were studied: a standard solution in a 50-50 methanol-acetonitrile mixture (for gas or liquid chromatography injection) which was to be diluted 1:10 in the laboratories' injection conditions, a spiked and a nonspiked natural mineral water, a raw water and a treated water. The various formulations were used to test the various stages of analysis:

- the sample in the solvent was used to test the analytical system without taking the extraction phase into account;

- the mineral water was used to:

- test contaminant control with the non-spiked solution,

- verify the extraction protocol's effectiveness in relation to theoretical spiking values on an eigenmatrix with little organic matter,

- compare the data with those from the raw water;

- the treated water matrix was used to assess the impact of the sodium thiosulfate stabilisation protocol on values close to analytical systems performance.

The samples were prepared and sent on the same day. They were packaged in 1-L brown glass flasks and did not undergo stabilisation treatment with the exception of the treated water (350 $\mathrm{mg}$ sodium thiosulfate per litre). Extraction was to take place within $48 \mathrm{~h}$. after the samples were prepared. This timetable was followed in $85 \%$ of cases.

Sample pre-treatment and analysis methods were not imposed in order to assess the robustness of the various extraction and analysis techniques on the four matrix types 


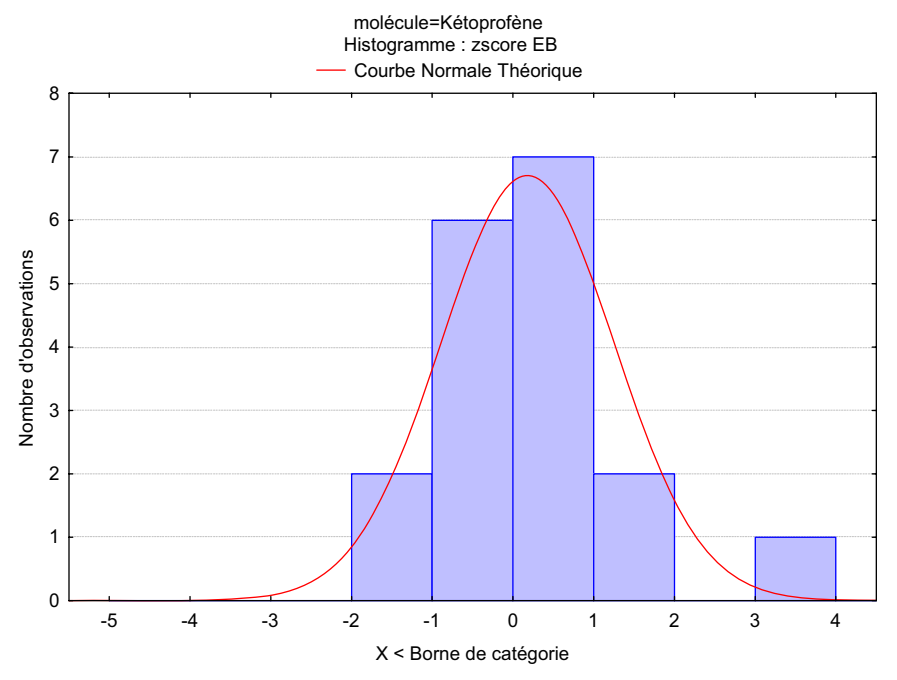

Fig. 1. Histogram of Z-scores for ketoprofen in raw water.

(solvent, mineral water, raw water and treated water). However, the laboratories were instructed not to filter the samples before analysis. The laboratories could also implement several analysis methods and submit several results for the same compound. One laboratory used three techniques (GC-MS/MS, LC-MS/MS and LC-MS/MS online preconcentration).

The analyses were to be performed in duplicate in repeatability conditions (2 extractions). Results below the limit of quantitation could be submitted, for example, in the following form: ' $<5 \mathrm{ng} / \mathrm{L}$ '.

\section{The results}

Statistical processing was performed in accordance with the procedure used by Ansès.

As a result, accuracy and precision information was obtained according to Mandel's $h$ and $k$ criteria. Robust tests were favoured when obtaining means and standard deviations so that all of the laboratories' data would be taken into account. No outliers were eliminated. The last step of statistical processing consisted in a study of individual performance in order to obtain results to calculate Z-scores, so as to identify 'compliant values', 'values to be monitored' and 'non-compliant values' for each compound-matrix pair.

These results can be used to assess a laboratory's performance in relation to the profession, which can be illustrated in the form of a histogram and a bell curve, as shown in Figure 1.

This figure shows the number of laboratories in each class. If we take the example of class [0-1], the histogram shows that 7 laboratories have a Z-score value (the laboratory's value minus the mean value divided by the standard deviation) between 0 and 1 .

The tolerance levels obtained through the Z-score calculation as described in Table 1 show that in the case of
Table 1. Z-score interpretation table.

\begin{tabular}{cc}
\hline Z-score $(z)$ & Interpretation \\
\hline$|z|=0.0$ & Performance corresponds to the assigned value \\
$0.0<|z| \leqslant 2.0$ & Performance is acceptable/satisfactory \\
$2.0<|z| \leqslant 3.0$ & Performance is questionable/needs improvement \\
$3.0<|z|$ & Performance is unacceptable/needs rectification \\
\hline
\end{tabular}

ketoprofen, $95 \%$ of the laboratories that submitted a raw water result had satisfactory performance.

Similar work was undertaken for the 12 compounds under study (17- $\alpha$-estradiol, 17- $\beta$-estradiol, ethinylestradiol, erythromycin, ofloxacin, tylosin, ibuprofen, diclofenac, ketoprofen, carbamazepine, atenolol, paracetamol) and the 5 samples.

The ILPT allowed each laboratory to compare its performance to other laboratories and to determine laboratory potential for possible standardisation and regulation in the current legislative corpus.

It is worthwhile to consider the extraction yields obtained by the laboratories against the performance criteria set out in the French Ministry of Health's Circular DGS/SD7A No. 2003-445 (2003) [1] which establishes 60-120\% tolerance levels on extraction yields of pesticides and similar products. When these values are applied to pharmaceutical products, Figure 2 shows 8 compounds that have extraction yields between 60 and $120 \%$. This interpretation is based on Tuckey diagrams of each compound. Note that quartiles 1 and 3 fall between 60 and $120 \%$. The methods that were used for two-thirds of the compounds studied in this ILT complied with the Ministry of Health's Circular. We can also illustrate that based on the same criteria, the methods used for $50 \%$ of the compounds had yields greater than $80 \%$.

The laboratories' protocol descriptions were used to draw up a diagram of analytical phases on the basis of 4 influential factors: the sample's pre-treatment upon receipt, the extraction protocol, the storage method and the detection-quantitation method. Figure 3 shows the laboratories' main practices but does not take performance into account since there were not enough responses for each standard methodology to statistically process the results.

On the basis of the participants' results (mean and standard deviation), relative standard deviation (RSD)) were calculated for each compound and within each matrix, in accordance with French standard NF EN ISO 16140 from 2003 [2]. The profession's inter-laboratory uncertainty was obtained by applying the formula of expanded uncertainty. The results given in Table 2 illustrate uncertainty ranging from $47 \%$ to $157 \%$, where $6 \%$ of the data have uncertainty under $50 \%, 15 \%$ of the data have uncertainty ranging from $50 \%$ to $60 \%$, and $35 \%$ of the data have uncertainty greater than $100 \%$. There are at least two explanations:

- laboratories' progress in relation to the method (development, validation, undergoing accreditation, accredited) can cause significant variations in results;

- since there are no guides on the assaying of pharmaceutical products in water, laboratories adapt or develop 


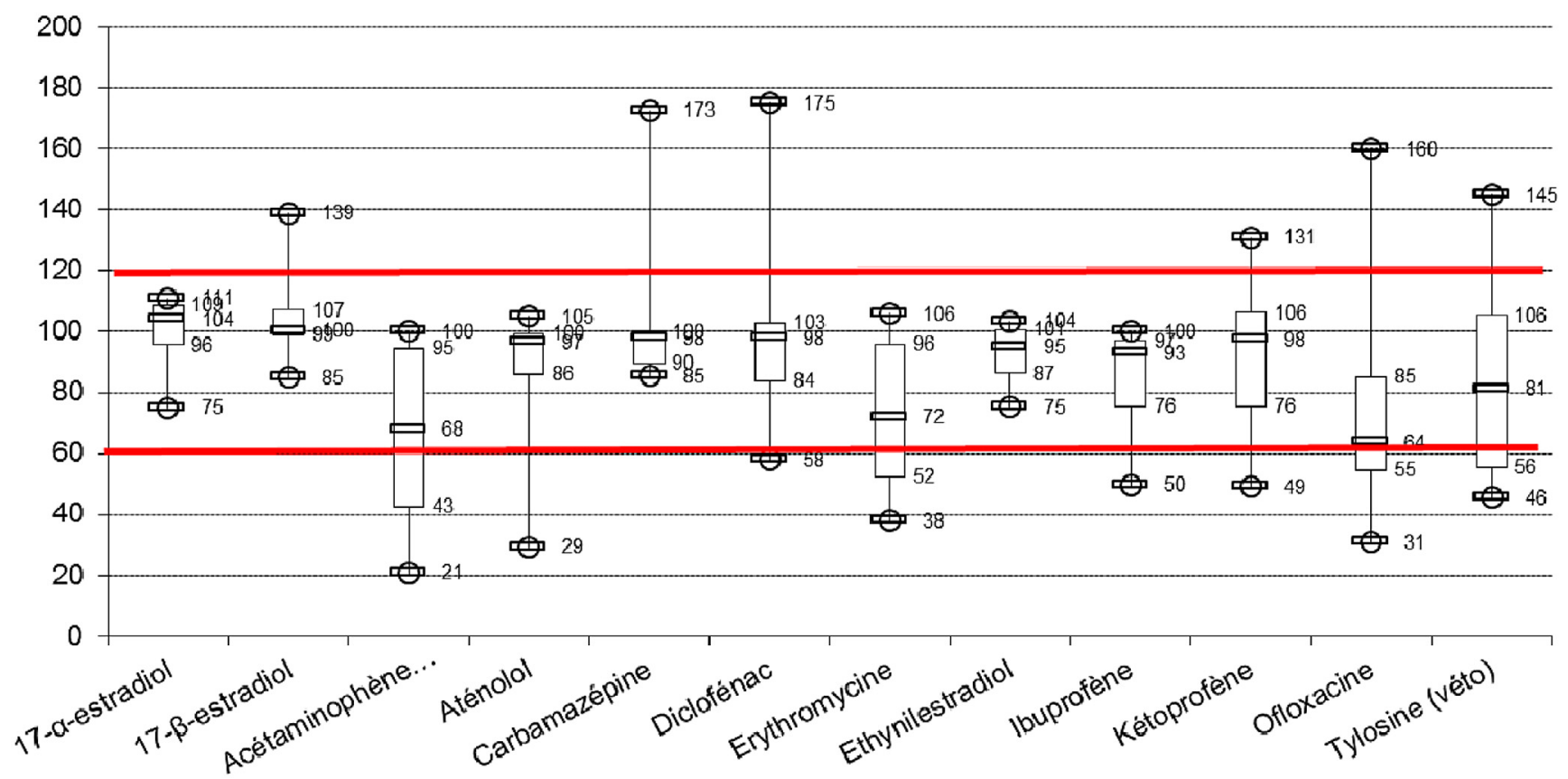

Fig. 2. Graphic illustration of yields according to the Tuckey diagram.

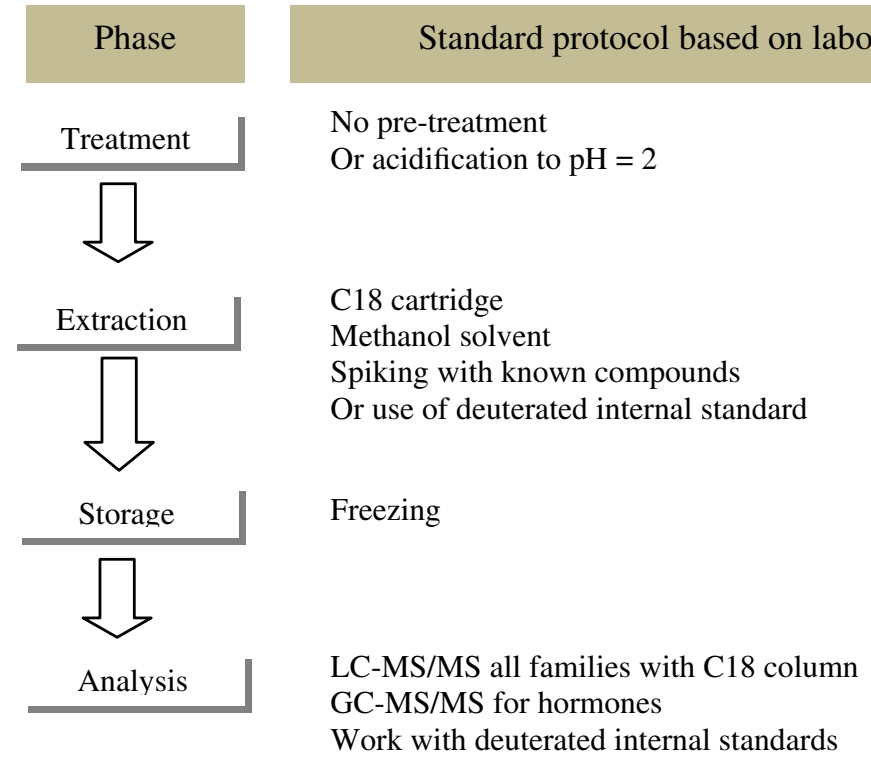

Fig. 3. Standard diagram of analytical phases on the basis of the information supplied by the laboratories.

their own protocol, even if a comparable technique has been used for analysing these compounds. This lack of consensus leads to biased results.

\section{Conclusion}

To conclude, this first French inter-laboratory test in the field of drinking water has generated a better view of current performance levels and laboratory practices. The latter appear to be fairly consistent from one laboratory to another.
The high level of participation shows the extent to which public and private laboratories are interested in these new analytical developments. The study of the comparability of results is especially important given that water prevalence campaigns are increasing in number.

This test also highlighted sensitive points such as the number of compounds studied compared to the number of compounds used in France (12 compounds studied/3000 compounds used in France). Inter-laboratory uncertainties indicated a high level of inter-laboratory performance variability which demonstrates a need to harmonise analytical practices. 
Table 2. Mean concentration and uncertainty by compound and by matrix.

\begin{tabular}{|c|c|c|c|c|c|c|c|c|c|}
\hline \multirow{2}{*}{ Family } & \multirow{2}{*}{ Molecules } & \multicolumn{2}{|c|}{ Standard solution } & \multicolumn{2}{|c|}{ Spike sample } & \multicolumn{2}{|c|}{ Tap water } & \multicolumn{2}{|c|}{ Groundwater } \\
\hline & & $\begin{array}{l}\text { Average } \\
(\mu \mathrm{g} / \mathrm{L})\end{array}$ & $\begin{array}{c}\text { Uncertainty } \\
(\%)\end{array}$ & $\begin{array}{c}\text { Average } \\
(\mu \mathrm{g} / \mathrm{L})\end{array}$ & $\begin{array}{c}\text { Uncertainty } \\
(\%)\end{array}$ & $\begin{array}{l}\text { Average } \\
(\mu \mathrm{g} / \mathrm{L})\end{array}$ & $\begin{array}{c}\text { Uncertainty } \\
(\%)\end{array}$ & $\begin{array}{l}\text { Average } \\
(\mu \mathrm{g} / \mathrm{L})\end{array}$ & $\begin{array}{c}\text { Uncertainty } \\
(\%)\end{array}$ \\
\hline \multirow{3}{*}{$\begin{array}{l}\text { Non-steroidal } \\
\text { anti-inflammatory }\end{array}$} & Diclofenac & 277 & 47 & 27.15 & 63 & 19.09 & 54 & 68.77 & 67 \\
\hline & Ibuprofen & 255 & 55 & 38.07 & 88 & 27.59 & 119 & 68.80 & 56 \\
\hline & Ketoprofen & 255 & 52 & 31.61 & 73 & 25.60 & 109 & 74.60 & 62 \\
\hline \multirow{3}{*}{ Antibiotics } & Erythromycin & 356 & 157 & 36.07 & 98 & 21.76 & 113 & 75.50 & 143 \\
\hline & Ofloxacin & 201 & 92 & 33.49 & 102 & 24.13 & 101 & 67.07 & 113 \\
\hline & Tylosin & 283 & 130 & 26.67 & 97 & 17.54 & 106 & 44.93 & 107 \\
\hline Beta-blockers & Atenolol & 239 & 101 & 29.09 & 56 & 14.80 & 93 & 59.10 & 79 \\
\hline Other & Paracetamol & 251 & 48 & 31.94 & 70 & 22.80 & 61 & 78.60 & 57 \\
\hline \multirow{3}{*}{ Hormones } & 17- $\alpha$-estradiol & 244 & 139 & 39.91 & 96 & 31.45 & 76 & 94.26 & 79 \\
\hline & 17 - $\beta$-estradiol & 277 & 100 & 36.22 & 108 & 25.35 & 75 & 86.40 & 88 \\
\hline & Ethynilestradiol & 234 & 121 & 30.91 & 90 & 27.29 & 70 & 81.54 & 47 \\
\hline Antipsychotics & Carbamazepin & 225 & 103 & 31.26 & 77 & 18.20 & 70 & 71.30 & 59 \\
\hline
\end{tabular}

This work will be followed by:

- inter-laboratory collaborative work in preparation for feedback and a standardised protocol that is scheduled to start in the second half of 2010 ;

- an estimate of intra-laboratory uncertainties to verify the compatibility of methods with Directive 2009/90/EC [3] in the framework of a forthcoming test.

Other inter-laboratory tests are planned, including a test on natural waters, which will be undertaken together with the AQUAREF network. Laboratories will be contacted in the second half of 2010 and the test will be conducted in the third quarter of 2011.

\section{References}

1. Circular DGS/SD7A No. 2003-445, of 17 September 2003, concerning the implementation terms of the French Order on water sample analysis methods and their performance characteristics

2. French Standard NF EN ISO 16140: 2003, Microbiology of food and animal feeding stuffs - Protocol for the validation of alternative methods

3. Commission Directive 2009/90/EC of 31 July 2009 laying down, pursuant to Directive 2000/60/EC of the European Parliament and of the Council, technical specifications for chemical analysis and monitoring of water status 